\title{
Community approaches to successfully address the hidden burden of sexually transmitted infections in rural South Africa
}

Remco P.H. Peters, Charlotte Hoffman, Jan Henk Dubbink, Nontembeko Mbambazela, Liteboho Maduna, Lucy Ranoto, Jean P. Railton, Marleen M. Kock, Helen E. Struthers, James A. Mclntyre

\section{Background}

- STI care in South Africa is provided at primary healthcare (PHC) facilities

- For various reasons, many individuals with STI symptoms do not access these services

- We used a community approach to mobilise individuals for STI care in rural South Africa

\section{Methods}

- We mobilised men and women with discharge and/or dysuria in rural Mopani District, South Africa, to visit their nearest healthcare facility

- The project was conducted at $6 \mathrm{PHC}$ facilities with a total catchment population of almost 65,000 people

- Mobilisation was done through: 1) posters at the healthcare facility, 2) community health workers (CHW), 3) empowered traditional leaders

- An expert team visited the facility on these two days to provide clinical care and ask individuals to participate in the study

- Vaginal swab and urine specimens was collected and tested for STIs and other reproductive tract infections

- Urine dipstick and culture was done in individuals reporting dysuria
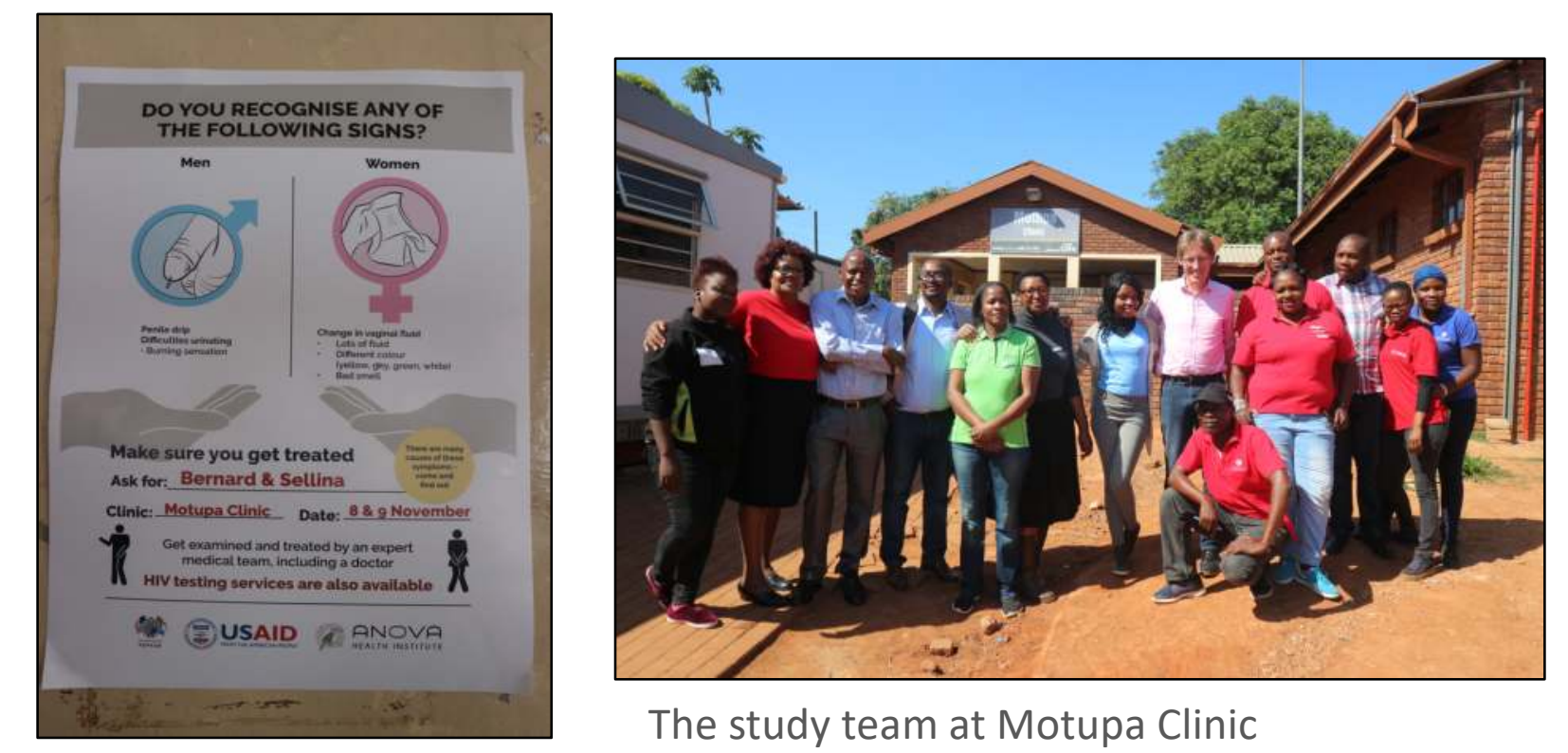

Poster used for mobilisation

\section{Further contact}

Prof R.P.H. Peters, Anova Health Institute

Email: rph.peters@gmail.com

\section{Results}

- We recruited 177 individuals (43 men and 134 women) mobilised to $6 \mathrm{PHC}$ facilities

- Individuals were mobilised through the clinic poster $(56 \%)$, the $\mathrm{CHW}(23 \%)$, a friend/relative $(18 \%)$ and traditional leader $(3.4 \%)$

- The unmet need for STI care is at least 1:275 for women and 1:650 for men in this population

- These individuals had not accessed STI care for a variety of reasons (Table 1 ).

\begin{tabular}{|lc|}
\hline Table 1. Reasons for not accessing services prior to mobilisation ( $\mathrm{n}=177)$ \\
\hline Main reason & Number \\
\hline Patient-related factors & $81(46 \%)$ \\
\hline Not aware of having these symptoms & $60(34 \%)$ \\
\hline No time or clinic too far away to consult & $4(2.3 \%)$ \\
\hline Embarrassed or afraid of reaction clinic staff & $12(6.7 \%)$ \\
\hline Traditional beliefs & $5(2.8 \%)$ \\
\hline & \\
\hline Clinical factors & $68(38 \%)$ \\
\hline Symptoms recurrent after treatment & $26(15 \%)$ \\
\hline Symptoms persistent despite treatment & $42(24 \%)$ \\
\hline & $28(16 \%)$ \\
\hline Healthcare service-related factors & $1(0.6 \%)$ \\
\hline Do not trust clinic staff (confidentiality) & $18(10 \%)$ \\
\hline Disappointed with health services previously & $9(5.1 \%)$ \\
\hline Lack of male healthcare workers & \\
\hline
\end{tabular}

- STIs were detected in $120(68 \%)$ of mobilised individuals: tests were positive for Chlamydia trachomatis (24\%), Neisseria gonorrhoeae (12\%), Trichomonas vaginalis $(56 \%)$ and Mycoplasma genitalium (9\%)

\section{Conclusion}

- There is a large unmet need for symptomatic STI care in this rural South African district

- A variety of patient-, individual- and servicerelated factors contribute to this hidden burden

- Prevalence of untreated STI is high among these symptomatic individuals 